\title{
Infant brain response to affective and discriminative touch: A longitudinal study using fNIRS
}

\author{
Helga O. Miguel, Óscar F. Gonçalves, Sara Cruz \& Adriana Sampaio
}

To cite this article: Helga O. Miguel, Óscar F. Gonçalves, Sara Cruz \& Adriana Sampaio (2019) Infant brain response to affective and discriminative touch: A longitudinal study using fNIRS, Social Neuroscience, 14:5, 571-582, DOI: 10.1080/17470919.2018.1536000

To link to this article: https://doi.org/10.1080/17470919.2018.1536000

View supplementary material $₫$

曲 Published online: 23 Oct 2018.

Submit your article to this journal

Шll Article views: 192

Q View related articles $\sqsubset$

View Crossmark data \lceil

Citing articles: 1 View citing articles $[7$ 


\title{
Infant brain response to affective and discriminative touch: A longitudinal study using fNIRS
}

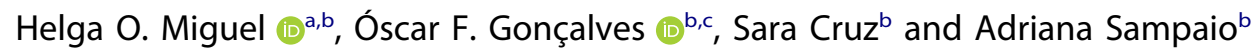 \\ aDivision of Imaging, Behavior, and Genetic Integrity, Section on Analytical and Functional, National Institute of Child and Human \\ Development, NIH, Bethesda, MD, USA; ' $N$ europsychophysiology Lab, CiPsi, School of Psychology, University of Minho, Campus de Gualtar \\ Braga, Portugal; 'Spaulding Neuromodulation Center, Spaulding Rehabilitation Hospital, Harvard Medical School, Charlestown MA, USA
}

\section{ABSTRACT}

The affective-motivational component of touch has been shown to consistently activate the social- brain network in children, adolescents and adults, including the posterior superior temporal sulcus (pSTS). However, very little is known about the neural mechanisms of affective touch processing during the first year of life. The objective of the present study was to analyze brain response to affective and discriminative touch in a sample of seven-month-old infants $(\mathrm{N}=35)$ who were followed longitudinally at 12 months of age $(\mathrm{N}=25)$. Infants were given affective and discriminative touch to the bare forearm while their brain response was recorded using functional near-spectroscopy (fNIRS). Seven-month-olds presented brain activation for affective and discriminative stimuli in channels placed over the somatosensory region, but no activation was recorded in channels placed in the temporal region for affective touch. At 12 months of age, infants presented a significant increase in hemodynamic activity in channels placed over the temporal region for affective touch, compared to seven-month-olds. Our study presents evidence of a developmental trajectory for distinct aspects of touch brain processing in the first year of life, with the recruitment of the temporal region for the affective component of touch, maturing in the second semester of life.

\section{ARTICLE HISTORY}

Received 13 April 2018

Revised 9 August 2018

Published online 24 October 2018

\section{KEYWORDS}

Touch processing; infancy; social-emotional development

\section{Introduction}

Touch is one of the most developed sensory systems at birth. Behavioral responses to touch can be observed as early as 7 weeks of gestation when the fetus moves after being touched on the lips (Hooker, 1952), or a few weeks later when the grasping and rooting reflexes emerge (Fifer \& Moon, 2003; Humphrey, 1964). Compared to other sensory modalities, namely vision and audition, touch receptors are matured and functionally developed in a newborn (see Bremner \& Spence, 2017; Gallace \& Spence, 2010 for reviews). Touch is the primary channel of communication between the mother and the newborn (Field, 2001; Hertenstein, Verkamp, Kerestes, \& Holmes, 2006), conveying soothing and comforting experiences during the first months of life (Jean \& Stack, 2012; Jean, Stack, \& Fogel, 2009). However, in contrast to the importance of touch early on in development, very little is known about how the brain processes touch during infancy.

Cutaneous information is sent to the central nervous system by a variety of tactile receptors. Low-threshold mechanoreceptors (LTMs) transduce information regarding properties of touch that include pressure, vibration, slip, and texture, and are instrumental in performing discriminative functions in everyday life such as handling objects and using tools (c.f., Abraira \& Ginty, 2013; McGlone, Vallbo, Olausson, Loken \& Wessberg, 2007; McGlone, Wessberg, \& Olausson, 2014). These receptors are innervated by myelinated $A \beta$ fibers that enable fast conduction of the nerve impulses, needed to quickly detect, discriminate and identify external stimuli (McGlone et al., 2014). LTMs are present in the entire body, but especially dense in the glabrous skin of the hand, particularly the digits (Kandel, Schawartz, \& Jessell, 2000; Mountcastle, 2005). C low-threshold mechanoreceptors (C-tactile afferents or $(\mathrm{CTs})$, present only in hairy skin, are responsible for transducing information regarding slow touch (Abraira \& Ginty, 2013). Microneurography experiments in humans have shown that these fibers are maximally activated by stimuli that move slowly, with maximum firing at velocities between $1-10 \mathrm{~cm}^{-1}$ (Essick et al., 2010; Loken, Wessberg, Morrison, McGlone, \& Olausson,

CONTACT Helga O. Miguel helga.miguel@nih.gov Division of Imaging, Behavior, and Genetic Integrity, Section on Analytical and Functional Bioophotonics, National Institute of Child Health and Human Development, 49 Convent Drive, Room 5A75, Bethesda MD 20892 
2009; H. Olausson, Wessberg, Morrison, McGlone, \& Vallbo, 2010). CT fibers are related to stimulus (pleasantness/unpleasantness) valence encoding and carry an important role in social behavior (Morrison, Loken, \& Olausson, 2010).

In contrast to the abundant research regarding $A \beta$ fibers (Mountcastle, 2005), only recently have CT fibers started gaining attention in neuroscience research. It has been proposed that these fibers are functionally distinct, and sub serve different aspects of touch behavior. Whereas $A \beta$ are responsible for the sensory-discriminative aspects of touch, CT fibers are hypothesized to be involved in affective-motivational behaviors (McGlone et al., 2007; McGlone et al., 2014; Morrison et al., 2010). At a cortical level, affective touch activates a brain network that includes the posterior insula, posterior superior temporal cortex (pSTS), medial prefrontal cortex (mPFC), and dorsal anterior cingulate cortex (dACC), along with the primary somatosensory cortex (S1) and secondary somatosensory cortex (S2) (Gordon et al., 2013; Olausson et al., 2002, 2008; Voos, Pelphrey, \& Kaiser, 2013). This network (insular cortex, pSTS, mPFC, dACC) is activated following stimuli that are social-emotional in nature. In neuropathy patients who lack $A \beta$ afferents (i.e., are not able to sense when they were touched), pure CT stimulation evoked activation in the dorsal posterior insular cortex, but not somatosensory areas S1 and S2. By contrast, pure $A \beta$-fiber stimulation (glabrous skin in healthy controls) activated S1 and S2, as did a combined stimulation of $A \beta$ and $C T$ stimulation to the forearm. These findings suggest that the CT system does not provide discriminative aspects of touch and probably holds distinct connections to cortical areas (Olausson et al., 2008, 2002).

Studies on the developmental trajectory of affective touch suggest that an extensive brain network, including the posterior insula, pSTS, medial prefrontal cortex, dACC, and also S1 and S2, is already in place in childhood during touch processing tasks (Bjornsdotter, Gordon, Pelphrey, Olausson, \& Kaiser, 2014; Van de Winckel et al., 2013). Additionally, there is evidence that the S2 continues to mature with age (Bjornsdotter et al., 2014). In infancy, the few studies conducted in the subject are somehow inconclusive. Whereas some studies failed to find activation in social brain regions before ten months of age in response to affective touch (Kida \& Shinohara, 2013; Miguel, Lisboa, Gonçalves, \& Sampaio, 2017), others were able to find brain activity in response to the same stimuli as early as one month of age (Jönsson et al., 2017; Tuulari et al., 2017).
Kida and Miguel's study used functional near infrared spectroscopy (fNIRS) to measure brain activity in response to different types of touch (affective versus discriminative). Kida and Shinohara (2013) looked at activation in the anterior prefrontal cortex and found that ten-month-old infants, but not three- or sixmonth-olds, showed an activation to affective touch, but not to discriminative touch. This study was limited by the few number of fNIRS channels used (two) and the fact that affective stimuli was delivered to the palm of the hand (lacking hairy skin), not specifically targeting CT fibers (Bennett, Bolling, Anderson, Pelphrey, \& Kaiser, 2014; Bjornsdotter et al., 2014; Gordon et al., 2013). The study by Miguel et al. (2017) looked at seven-month-olds' brain activation to affective and discriminative touch in a primary sensory region (SI) that processes both affective and discriminative stimuli, and an associative region (pSTS) known to process only affective touch. Interestingly, infants presented greater hemodynamic function in the channels placed over the somatosensory region for the two types of stimuli, but no activation was found over the right pSTS. Nevertheless, the conclusions from these studies are limited to superficial cortical regions of the brain, since fNIRS only provides the spatial resolution of about $1 \mathrm{~cm}^{2}$.

In contrast, the findings from Jönsson et al. (2017) and Tuulari et al. (2017) suggest that the affective touch system is functional at very early stages of postnatal life. Both studies found significantly greater activation to CT targeted touch in the insular cortex, suggesting that the network responsible for detecting pleasantness from tactile stimuli is already in place when we are born. In addition, both studies found activation in the superior temporal region, but to a lesser extent (uncorrected threshold), suggesting that the response to slow stroking might follow a developmental trajectory from deeper brain regions of the cortex, namely the insular cortex, to more superficial regions, as with the posterior temporal sulcus. This would be consistent with the fact that brain regions responsible for sensory motor functions mature earlier in development, whereas associative high-order regions, including the temporal lobe, mature later (Lenroot \& Giedd, 2006).

Given the inconclusive findings for the brain mechanisms that underlie the processing of affective touch in infants and the maturation evidenced in the second semester of life, namely social behaviors including gaze following (Tomasello, Carpenter, \& Liszkowski, 2007) and emotional face processing (Leppanen \& Nelson, 2009), we selected a group of seven-month-old infants and followed them longitudinally until 12 months of age in order to understand how the brain evolves in response 
to affective touch. fNIRS was used to measure brain activity in response to $C T$ stimulation (affective touch) and $A \beta$ stimulation (discriminative touch), focusing on two distinct regions of interest, the STS and the somatosensory cortex. The STS was chosen because it has been reported to activate in response to $C T$ targeted stimuli using fNIRS (Bennett et al., 2014) and is a brain region associated with earlier processing of social cues like biological motion and face or voice processing. The somatosensory cortex was selected because it is a wellresearched primary sensory region that activates in response to tactile stimuli in infants (Verriotis et al., 2016). We hypothesized that both seven- and 12month-old infants would present brain activity in the somatosensory cortex, for discriminative and affective touch. Additionally, we also hypothesized increased activity for affective touch in pSTS for 12-month-olds, but not for seven-month-olds.

\section{Methods}

\subsection{Participants}

Infants were recruited from local parenting classes, social networks, and daycare centers. Thirty-five infants at seven months of age were included in the study (mean age $228.77 \pm 89.19$ days, range $214-244 ; 14$ females), and all but one were born full term (mean time in weeks $39.07 \pm 1.22$, range 36.6-41) with a normal birth weight (>2750 g, except for one infant who weighted $2350 \mathrm{~g}$ ). Initially, 49 infants were tested, but fourteen of the initial infant sample were excluded due to fussiness $(n=2)$ or not undergoing the minimum number of three acceptable trials to be included $(n=12)$, leaving 35 viable infants in the empirical sample. From the viable sample of 49 infants (all who met the selection criteria), 25 12-month-old infants were included in the second phase of the longitudinal study (mean age $387.15 \pm 9.33$ days, range 372-406). Twenty additional infants were tested but excluded due to fussiness ( $n=4$ ), not undergoing the minimum number of three acceptable trials to be included $(n=7)$, motion artifact and/or noisy data $(n=7)$, and experimental error $(n=2)$. In addition, 4 infants were not present at the follow-up visit. The total attrition rate was $29 \%$ for the seven-month-olds and $45 \%$ for the 12 month-olds, which is comparable to other NIRS studies in infancy (Lloyd-Fox, Szeplaki-Kollod, Yin, \& Csibra, 2015). Infant demographics and developmental data are shown in Table 1. Demographics refer to infants included in the final samples at seven and 12 months of age. Mothers signed an informed consent prior to the start of the experiment. The experimental protocol was approved by the local ethics committee.

\subsection{Stimuli}

Two stimuli were used to represent the affective and discriminative dimensions of touch. The affective stimuli incorporated a $7 \mathrm{~cm}$ wide watercolor brush (Bennett et al., 2014; Kaiser et al., 2016) and the discriminative stimuli a squared-shape piece of wood $2 \times 2 \mathrm{~cm}$ (Kida \& Shinohara, 2013). Affective stimuli comprised slow strokes $(8 \mathrm{~cm} / \mathrm{s})$ with the watercolor brush, administered in a proximaldistal direction by a trained experimenter (Triscoli, Olausson, Sailer, Ignell, \& Croy, 2013). This velocity was used in previous experiments and has been shown to target CT fibers (Loken et al., 2009). Discriminative stimuli consisted of applying pressure with the wood block in the dorsal forearm in a proximal-distal direction for the same period. To parallel the same distance of the brush, the wood block was applied three times a second (between 21 and 24 stimuli applied). The discriminative stimuli did not include any stroking movement, assuring that the fibers stimulated were the $A \beta$ fibers.

Both discriminative and affective stimuli were delivered to the right dorsal forearm of the infant (bare arm) in a within-subject block design procedure resulting in two alternating blocks of each experimental condition (affective, discriminative) of eight trials each (Bennett et al., 2014). One trial consisted of ten seconds of stimulation followed by a baseline period of 20 seconds of rest. Stimuli were presented in an alternating way. Baseline stimuli (rest) consisted of the infant viewing a silent movie (Czech cartoon Krtecek) that played

Table 1. Sample demographics.

\begin{tabular}{lcc}
\hline Characterization of the sample & 7 months & 12 months \\
\hline Number of infants & 35 & 25 \\
Age at birth (weeks) & $39.07 \pm 1.22(36.6-41)$ & $39.167 \pm 1.167(37-41)$ \\
Age at study (days) & $228.77 \pm 9.20(214-244)$ & $387.15 \pm 9.33(372-406)$ \\
Female infants & 14 & 11 \\
Weight at birth $(\mathrm{g})$ & $3384.24 \pm 459.59(2350-4390)$ & $3245.96 \pm 457.27(2420-4390)$ \\
Height at birth $(\mathrm{cm})$ & $48.90 \pm 2.64(43-54)$ & $48.58 \pm 1.51(44-52.90)$ \\
Cesarean deliveries & $7(20 \%)$ & $8(32 \%)$ \\
Apgar 1 & $9.33 \pm 1.10(5-10)$ & $9.33 \pm .96(6-10)$ \\
Apgar10 & $9.96 \pm .19(9-10)$ & $9.96 \pm .20(9-10)$ \\
\hline
\end{tabular}


continuously throughout the session and simultaneously with the task, as used previously in research (Fairhurst, Loken, \& Grossmann, 2014). See Figure 1 for a schematic representation of the paradigm.

\subsection{NIRS recording}

Hemodynamic responses were recorded using the UCLfNIRS topography system (Everdell et al., 2005) with 12 sources and six detectors using two continuous wavelengths of source light at 780 and $850 \mathrm{~nm}$. Data were sampled every $100 \mathrm{~ms}(10 \mathrm{~Hz}$ ) (for a detailed description of fNIRS methodology see Lloyd-Fox, Blasi, \& Elwell, 2010).

NIRS data were recorded from 18 channels, nine of which were placed over the left somatosensory region and nine placed over the right pSTS (see Figure 2). The NIRS probe was customized for this experiment using an elastic cap (Easy Cap) with reference to the 10-5 system (see Figure 2(b)) (Jurcak, Tsuzuki, \& Dan, 2007). Depending on the head circumference at seven- and at 12 months of age, caps for different head-

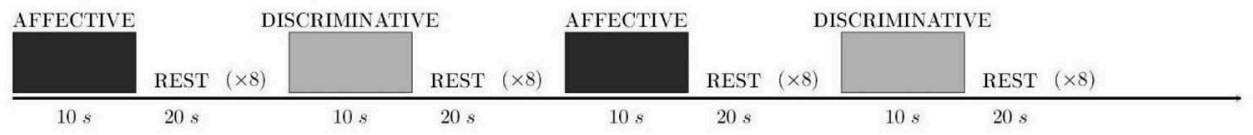

Figure 1. Experimental design. Each block consisted of a tactile stimulus delivered for ten seconds, followed by 20 seconds of rest, repeated eight times. There was a total of two blocks per condition (two affective + two discriminative).
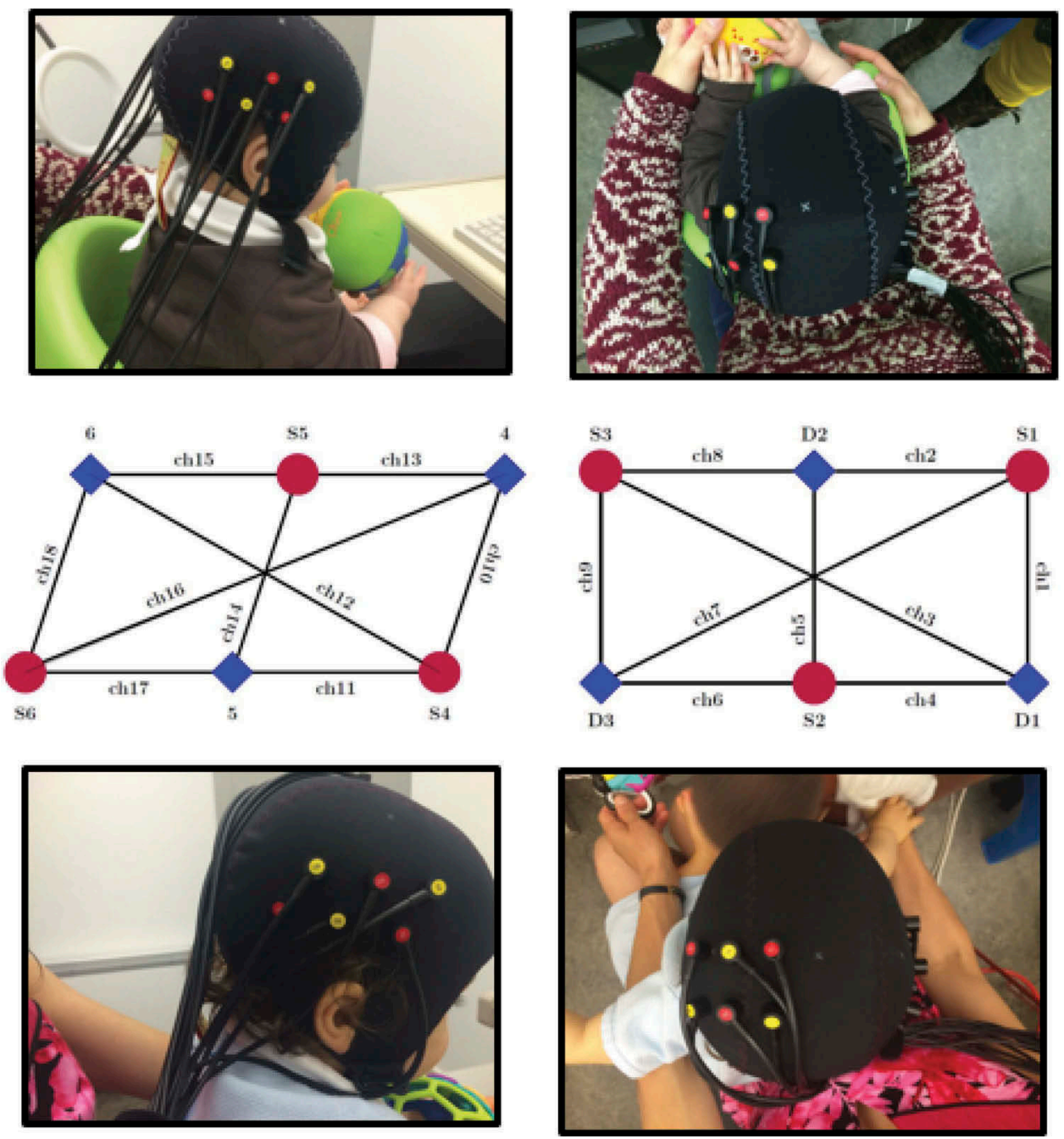

Figure 2. NIRS data were recorded from 18 channels: nine placed over right pSTS (left panel) and nine placed over the left somatosensory region (right panel). The top panel depicts the fNIRS headgear on a seven-month-old and the bottom panel on a 12month-old. Red circles represent sources and blue squares represent detectors. The NIRS probe was customized to this experiment using an elastic cap (Easy Cap), with reference to the 10-5 system. 
circumferences were used ( 44 and $46 \mathrm{~cm}$ ). Inter-optode distance was $22 \mathrm{~mm}$ in the pSTS region (except for the two longest channels that crossed the middle of the array and were around $45 \mathrm{~mm}$ ) and between 40 and $45 \mathrm{~mm}$ in the somatosensory region. Before the experiment, measurements of head circumference and nasion-inion were taken to align the headgear with the 10-5 system (see Table 1 for head measurements). The cap was placed centrally in the top of $\mathrm{Cz}$, with channel 11 (correspondent to TP8) placed above the peri-auricular point.

\subsection{Procedure}

While the mother completed the consent form and the experimenter completed the demographic questionnaire, infants were allowed to familiarize themselves with the setting and play with toys in the experimenter's room. During this time, infants were held in their mother's lap and head measurements were taken. An experimenter continued to play with the infant to direct his/her attention away while the cap was being placed. The baby was then seated in a baby seat (Jelly Mom Baby Chair) to avoid physical contact with the mother, and the silent movie was started (Czech cartoon Krtecek as in Fairhurst et al., 2014).

The infant was seated at approximately $70 \mathrm{~cm}$ away from a computer screen (screen size: $53^{*} 30$ ). The experimenter sat to the back right-hand side of the infant and parent, administered the stimuli, and re-directed the infant's attention to the screen when needed. No visual contact took place between the experimenter and the infant during the course of the experiment. Parents were instructed to avoid any interaction with the infant, unless he/she became fussy. The experiment took place in a dimmed-light room to avoid light-interference. Breaks were introduced when needed to keep the infant involved throughout the experiment. The experiment ended when the infant completed the four blocks or when he/she became fussy. All the sessions were videotaped for offline coding.

\subsection{Data processing and analysis}

The videos from the individual sessions were coded offline by an observer who was blind to the inclusion criteria. Each participant engaged in a maximum of 16 trials per experimental condition. Participants were only included if they successfully completed at least three quality trials (Lloyd-Fox, Wu, Richards, Elwell, \& Johnson, 2015). To be considered an acceptable trial, the following criteria needed to be met: (1) the infant did not move the arm in any direction while the stimulus was being administered; (2) the infant was not looking at the experimenter or the mother while the stimulus was being administered; (3) the infant did not touch the experimenter or the mother while the stimulus was being delivered. Seven-month-olds completed an average of $6.65 \pm 3.06$ affective trials (range $3-14$ ) and $7.25 \pm 2.82$ (3-17) discriminative trials; 12-month-olds completed an average of $6.68 \pm 2.15$ affective trials (range $4-13$ ) and $6.32 \pm 2.52$ (3-12) discriminative trials. No statistical difference was found for the number of trials completed at seven $\left(X^{2}=119.79, p=0.09\right)$ nor at 12 months of age $\left(x^{2=} 31.39, p=0.885\right)$.

Hemodynamic activity data was processed using HOMER2 (MGH - Martinos Center for Biomedical Imaging, Boston, MA, USA), a MATLAB (The MathWorks, Inc., Natick, MA, USA) software package. The attenuated light intensities measured by the detecting optodes were converted to optical density units and assessed for movement artifact using Principal Component Analysis (PCA) set at 0.9. (Cooper et al., 2012). Both a significant increase in oxy-hemoglobin $\left(\mathrm{HbO}_{2}\right)$ or a decrease in deoxy-hemoglobin ( $\mathrm{Hbb}$ ) are accepted as indicators of hemodynamic activity and were used for data analysis. Since we only included trials in which the infant was not moving his/her arm, rejecting trials (PCA) is a preferable method over correction (e.g., wavelet motion correction). Data were then low-pass filtered at .5 (Lloyd-Fox et al., 2015) and used to calculate the change in concentration of the hemoglobin chromophore according to the modified Beer-Lambert Law (Delpy et al., 1988), assuming a pathlenght factor of 5.13 (Duncan et al., 1995). Traces were segmented into 30 second epochs, starting two seconds prior to each stimulus. The baseline corresponded to the mean time from -2 to 0 , as in previous fNIRS studies (Ravicz, Perdue, Westerlund, Vanderwert, \& Nelson, 2015).

Statistical analysis was performed using SAS (Statistical Analysis Software) 9.4v. Grand average haemodynamic response $(\mu \mathrm{m})$ was calculated for the two conditions (affective and discriminative). For each channel, the maximum change in $\mathbf{H b O}_{\mathbf{2}}$ (increase in chromophore concentration) and $\mathbf{H b b}$ (decrease in chromophore concentration) in response to each experimental condition was assessed relative to the baseline using a mixed-effect regression model. After visual inspection of the grand mean concentration changes of each chromophore for each condition, we realized there were differences in the latency of the response; for this reason, and also to include the maximum signal changes of both stimuli, five-time windows were determined for the analyses: $\mathrm{t} 1=0$ to five seconds; t2 = five to ten seconds; t3: ten to 15 seconds; t4: 15 to 20 seconds and t5: 20 to 25 seconds. The variable 
time was dummy coded to capture non-linear relation over time. This preliminary analysis allowed us to determine that the long-channels (diagonal channels three, seven, 12 and 16 in Figure 2) resulted in noisy data and, therefore, were excluded from the analysis. We computed linear mixed-models separately for each of the remaining channels to test the differences between condition ( 2 levels), time ( $1-5$ levels), and age ( 2 levels: seven and 12 months of age), on $\mathrm{HbO}_{2}$ and $\mathrm{Hbb}$ concentrations. The variable time was dummy coded to capture non-linear relations between $\mathrm{HbO} 2$ concentration and stimuli over time. Reported $p$ values were not adjusted for type-l error rates.

\section{Results}

We first describe the results separately for seven- and 12-month-old infants (discriminative and affective touch against baseline). We then present the contrasts between the two age groups for the different conditions.

\subsection{Effects of condition against baseline}

\subsubsection{Seven-month-old infants}

Discriminative touch. To assess the response to discriminative stimuli for each channel, the brain's hemodynamic response to the discriminative condition (wood block) was analyzed relative to a baseline (the silent movie). This analysis revealed a significant increase in $\mathrm{HbO} 2$ centered over the somatosensory region for channel one at times 3 $(\mathrm{t}(1,205)=12.91, p<0.001)$ and $4(\mathrm{t}(1,205)=7.21$, $p=0.008)$, channel two at time 3 (t $(1,205)=6.52$, $p=0.011)$ and channel five at time $3(\mathrm{t}(1,205)=4.56$, $p=0.034)$. Marginally significant increases were found for channel eight at time $3(t(1,205)=3.04, p=0.08)$. Over the pSTS region, discriminative touch elicited a significant increase in channel 13 at times $2(\mathrm{t}(1,205)=9.84$, $p=0.002)$ and 3 (t $(1,205)=15.75, p<0.001)$, and in channel 15 at times $2(\mathrm{t}(1,205)=8.65, p=0.004)$ and $3(\mathrm{t}(1$, $205)=5.07, p=0.025)$. Significant $\mathrm{Hbb}$ decrease was also observed in channels 1 at times $2(\mathrm{t}(1,205)=-2.42$, $p=0.016), 3$ (t $(1,205)=-3.43, p<0.001)$ and $4(\mathrm{t}(1,205)=-2.07, p=0.039)$, channel 2 at time $2(\mathrm{t}(1,205)=-2.22, p=0.028)$ and $3(\mathrm{t}(1,205)=-2.11$, $p=0.036)$, channel 8 at time $2(\mathrm{t}(1,205)=-1.98, p=0.048)$ and $3(\mathrm{t}(1,205)=-2.52, p<0.012)$, and channel 9 at time 3 (t $(1,205)=-2.06, p=0.041)$. Over the pSTS region, significant $\mathrm{Hbb}$ decrease was observed in channel 14 at time $1(\mathrm{t}(1,205)=-1.97, p=0.050)$ and channel 15 at time 2 ( $\mathrm{t}(1,205)=-2.43, p=0.016)$. Additionally, marginally significant results were found for channel 2 at times $4(\mathrm{t}(1,205)=-1.81, p=0.072)$ and $5(\mathrm{t}(1,205)=-1.83$, $p=0.069)$, channel 13 at time 2 (t $(1,205)=-1.83$, $p=0.069)$ and channel 14 at time $1(\mathrm{t}(1,205)=-1.92$, $p=0.056$ ). See Tables S1 and S2 in the supplementary materials for channel-by-channel contrasts.

Affective touch. Hemodynamic response for the affective stimuli resulted from the contrast between the affective stimuli (brush) relative to a baseline. Analyses for each channel revealed a significant hemodynamic increase in $\mathrm{HbO} 2$ for channel one at times $4(\mathrm{t}(1,205)=5.88, p=0.016)$ and $5(\mathrm{t}(1,205)=4.50$, $p=0.035)$. Significant $\mathrm{Hbb}$ decrease was also observed in channel 10 at time $3(\mathrm{t}(1,205)=-2.03, p=0.044)$, and channel 18 at time $4(t(1,205)=-2.22, p=0.027)$. Additionally, marginally significant $\mathrm{Hbb}$ decrease was also found for channel 1 at time $3(t(1,205)=-1.79$, $p=0.075)$ and channel 17 at time $4(\mathrm{t}(1,205)=-1.74$, $p=0.084)$. See Tables $\mathrm{S} 1$ and $\mathrm{S} 2$ in the supplementary materials for channel-by-channel contrasts.

\subsubsection{Twelve-month-old infants}

Discriminative touch. For discriminative touch, there was a significant hemodynamic increase in $\mathrm{HbO} 2$ centered over the somatosensory region for channel six at times 2 ( $\mathrm{t}(145)=2.25, p=0.025)$ and $3(\mathrm{t}(145)=2.15$, $p=0.033)$. In addition, marginally significant hemodynamic increases in $\mathrm{HbO} 2$ were found for channels four $(\mathrm{t}(145)=p=0.06)$ and five $(\mathrm{t}(145)=3.25, p=0.070)$ at time 3. Over the pSTS region, there was a significant hemodynamic increase of $\mathrm{HbO}_{2}$ at time 4 for channel 17 (t $(145)=4.04, p=0.040$ ). See Table 2 for $\mathrm{HbO}_{2}$ channel-by-channel contrasts. Significant $\mathrm{Hbb}$ decrease was observed in channel 4 at time $3(\mathrm{t}(145)=-2.08$, $p=0.039)$ and channel 5 at time 3 (t $(145)=-1.97$, $p=0.050$ ). See Table S3 in supplementary materials for $\mathrm{Hbb}$ channel-by-channel contrasts.

Affective touch. Hemodynamic response for the affective stimuli showed a significant hemodynamic $\mathrm{HbO} 2$ increase in channel one at time 4 (t $(145)=4.82$, $p=0.029$ ), in channel two times3 (t $(145)=7.33$, $p=0.007)$ and $4(\mathrm{t}(145)=3.83, p=0.052)$, and in channel nine at time4 ( $\mathrm{t}(145)=11.70, p<0.01)$. In addition, marginally significant hemodynamic increases in $\mathrm{HbO} 2$ were found in channel one at time 3 (t $(145)=2.92, p=0.089$ ) and in channel nine at time 5 ( $\mathrm{t}(145)=3.17, p=0.079$ ). Over the PSTS region there was a significant hemodynamic increase of $\mathrm{HbO} 2$ for channel ten at times 2 ( $\mathrm{t}(145)=5.13$, $p=0.025)$ and 3 (t $(145)=9.39, p=0.003)$, channel 11 at time 1 ( $\mathrm{t}(145)=5.26, p=0.023)$, channel 13 at times 3 (t $(145)=10.08, p=0.002)$ and $4(\mathrm{t}(145)=4.69$, $p=0.032)$, channel 14 at time $4(\mathrm{t}(145)=5.86, p=0.017)$, and channel 15 at time 3 (t $(145)=6.60, p=0.011$ ) and $4(\mathrm{t}(145)=5.51, p=0.020)$. In addition, marginally significant responses were also found for channel 11 at time 
Table 2. Significant $\mathrm{HbO}_{2}$ responses to different contrasts between discriminative and affective stimuli at $12 \mathrm{M}$.

\begin{tabular}{|c|c|c|c|c|c|c|c|c|c|}
\hline \multicolumn{5}{|c|}{ Discriminative $>$ Affective } & \multicolumn{5}{|c|}{ Affective $>$ Discriminative } \\
\hline Channel & Time Window & $\beta$ & SE & $p$ value & Channel & Time Window & $\beta$ & SE & $p$ value \\
\hline \multirow[t]{6}{*}{6} & $\begin{array}{l}2 \\
3\end{array}$ & 0.044 & 0.012 & $<0.010$ & 9 & 4 & 0.048 & 0.013 & $<0.001$ \\
\hline & & & & & 10 & 3 & 0.037 & 0.017 & 0.030 \\
\hline & & & & & 14 & 4 & 0.038 & 0.011 & $<0.001$ \\
\hline & & & & & 15 & 4 & 0.050 & 0.016 & 0.010 \\
\hline & & & & & 17 & 3 & 0.038 & 0.011 & $<0.001$ \\
\hline & & & & & & 4 & 0.038 & 0.011 & $<0.001$ \\
\hline
\end{tabular}

a time window 1: 0-5 seg; time window 2: 5-10 seg; time window 3: 10-15 seg; time window 4: 15-20 seg.

${ }^{\mathrm{b}}$ Channels six and nine are located in somatosensory region; Channels ten, 14,15 e 17 are located in temporal region.

$2(\mathrm{t}(145)=3.62, p=0.060)$. See Table 2 for $\mathrm{HbO}_{2}$ channelby-channel contrasts. Significant $\mathrm{Hbb}$ decrease was found for channel 1 at times $3(\mathrm{t}(145)=-3.39, p<0.001)$ and $4(\mathrm{t}$ $(145)=-3.10, p=0.002)$, channel 9 at times $3(\mathrm{t}(145)=-2.09, p=0.038)$ and $4(\mathrm{t}(145)=-3.42$, $p<0.001)$, channel 13 at time 4 (t $(145)=-2.99$, $p=0.003$ ), and channel 15 at time 4 (t $(145)=-2.01$, $p=0.05)$. In addition, marginally significant results were found for channel 1 at time 5 ( $\mathrm{t}(145)=-1.75, p<0.081$ ). See Table S3 in supplementary materials for Hbb channelby-channel contrasts.

\subsection{Effect of condition and block}

For the discriminative $>$ affective contrast in channels placed over the somatosensory region, channel six showed a significant $\mathrm{HbO} 2$ increase for the discriminative stimuli compared to affective stimuli at times $2(\mathrm{M}=0.044, \mathrm{SE}=0.012 ; \mathrm{t}(304)=3.77, p<0.01)$ and $3(\mathrm{M}=0.030, \mathrm{SE}=0.012 ; \mathrm{t}(304)=2.59, p=0.01)$. No significant differences were found for $\mathrm{Hbb}$ decrease in channels placed over the somatosensory region. For the affective $>$ discriminative contrast in channels placed over the somatosensory region, channel nine showed significant $\mathrm{HbO} 2$ increase $(\mathrm{M}=0.048, \mathrm{SE}=0.013$; $\mathrm{t}(188)=1.79, p<0.01)$. In addition, significant $\mathrm{Hbb}$ decrease was found for channel 1 at time $3(M=0.034$, $\mathrm{SE}=0.015 ; \mathrm{t}(304)=2.24, p=0.026)$ and time $4(\mathrm{M}=0.039, \mathrm{SE}=0.015 ; \mathrm{t}(304)=2.58, p=0.01)$. In the pSTS region, affective touch resulted in a greater $\mathrm{HbO}_{2}$ increase for channel ten at time $3(\mathrm{M}=0.037$, $\mathrm{SE}=0.017 ; \mathrm{t}(304)=2.18, p=0.03)$, channel 14 at time 4 $(\mathrm{M}=0.038, \mathrm{SE}=0.011 ; \mathrm{t}(188)=3.35, p<0.001)$, channel 15 at time $4(\mathrm{M}=0.050, \mathrm{SE}=0.016$; $\mathrm{t}(188)=3.15, p=0.01)$ and channel 17 at time $3(\mathrm{M}=0.038, \mathrm{SE}=0.011 ; \mathrm{t}(188)=3.35, p<0.001)$ and four $(\mathrm{M}=0.038$, SE $=0.011 ; \mathrm{t}(188)=3.35$, $p<0.001)$. Moreover, marginally significant activation was found for channel 9 at time $4(M=0.021$, $\mathrm{SE}=0.012 ; \mathrm{t}(304)=1.79, p=0.076) .15$ at time $3(\mathrm{M}=0.039, \mathrm{SE}=0.016 ; \mathrm{t}(304)=2.42, p=0.070)$. Significant decreases in $\mathrm{Hbb}$ were also found for channel 13 at time $4(\mathrm{M}=0.05, \mathrm{SE}=0.019 ; \mathrm{t}$ $(304)=2.54, p=0.012)$; channel 15 at time 3 $(\mathrm{M}=0.041, \mathrm{SE}=0.015 ; \mathrm{t}(304)=2.74, p=0.007)$ and $4(\mathrm{M}=0.041, \mathrm{SE}=0.015 ; \mathrm{t}(304)=2.78, p=0.006)$. See Table 2 for significant channel-by-channel $\mathrm{HbO}_{2}$ contrasts. See Table S3 in supplementary materials for significant channel-by-channel Hbb contrasts.

\subsection{Effect of age, condition, and block}

For the contrast discriminative $7 \mathrm{M}>$ discriminative $12 \mathrm{M}$, a significant $\mathrm{HbO}_{2}$ increase was found in channel two at times $3(\mathrm{M}=0.056, \mathrm{SE}=0.024 ; \mathrm{t}(536)=2.38, p=0.018)$ and $4(\mathrm{M}=0.053, \mathrm{SE}=0.024 ; \mathrm{t}(536)=2.25, p=0.025)$, channel five at time $4(\mathrm{M}=0.033, \mathrm{SE}=0.016 ; \mathrm{t}(536)=1.98$, $p=0.05)$, channel eight at time $3(\mathrm{M}=0.035, \mathrm{SE}=0.015$; t $(412)=2.35, p=0.019)$, and channel 13 at time $2(\mathrm{M}=0.035, \mathrm{SE}=0.014 ; \mathrm{t}(536)=1.94, p=0.053)$. Marginally significant results were found for channel eight at time $2(\mathrm{M}=0.033, \mathrm{SE}=0.015 ; \mathrm{t}(412)=2.27, p=0.088)$. Additionally, significant $\mathrm{Hbb}$ decrease was found for channel 4 at time $4(\mathrm{M}=0.047$, SE $=0.019 ; \mathrm{t}(536)=2.19$, $p=0.013$, and $5(\mathrm{M}=0.060, \mathrm{SE}=0.019 ; \mathrm{t}(536)=3.24$, $p=0.001)$, channel 5 at time $4(\mathrm{M}=0.042, \mathrm{SE}=0.013$; $\mathrm{t}(536)=3.18, p=0.002)$ and $5(\mathrm{M}=0.058, \mathrm{SE}=0.013$; $\mathrm{t}(536)=4.39, p<0.01)$, channel 10 at time $2(\mathrm{M}=0.036$, $\mathrm{SE}=0.016, \mathrm{t}(536)=2.3, p=0.022)$ and $4(\mathrm{M}=0.042$, $\mathrm{SE}=0.016$; $\mathrm{t}(536)=2.66, p=0.008)$, channel 15 at time $4(\mathrm{M}=-0.036, \mathrm{SE}=0.016, \mathrm{t}(536)=1.96, p=0.051)$. Marginally $\mathrm{Hbb}$ decrease was found in channel 2 at time $2(\mathrm{M}=0.033, \mathrm{SE}=0.019 ; \mathrm{t}(536)=1.69, p=0.092)$ and channel 8 at time $2(\mathrm{M}=0.021, \mathrm{SE}=0.012 ; \mathrm{t}(536)=1.72$, $p=0.087)$ and $3(\mathrm{M}=0.023, \mathrm{SE}=0.012 ; \mathrm{t}(536)=1.84$, $p=0.067$ ) No significant differences were found for the contrast for affective $7 \mathrm{M}>$ affective $12 \mathrm{M}$.

For the contrast discriminative $12 \mathrm{M}>$ discriminative $7 \mathrm{M}$, a significant $\mathrm{HbO}_{2}$ increase was found in channel six at time $1(\mathrm{M}=0.022, \mathrm{SE}=0.010 ; \mathrm{t}(304)=2.01, p=0.045)$, channel 14 at time $5(\mathrm{M}=0.036, \mathrm{SE}=0.014 ; \mathrm{t}(420)=2.52$, $p=0.012)$, and channel 17 at time $4(\mathrm{M}=0.055, \mathrm{SE}=0.020$; $\mathrm{t}(306)=2.66, p=0.008)$ and $5(\mathrm{M}=0.046$, $\mathrm{SE}=0.018$; t $(306)=2.54, p=0.012$ ). The contrast affective $12 \mathrm{M}$ 
$>$ affective 7M resulted in a significant increase of hemodynamic activity for channel eight at time $4(M=0.033$, $\mathrm{SE}=0.015 ; \mathrm{t}(412)=2.26, p=0.025)$, channel ten at time $3(\mathrm{M}=0.046, \mathrm{SE}=0.019 ; \mathrm{t}(536)=2.39, p=0.017)$, channel 11 at time $1(\mathrm{M}=0.058 \mathrm{SE}=0.024 ; \mathrm{t}(304)=2.41, p=0.016)$ and $2(\mathrm{M}=0.056 \mathrm{SE}=0.024 ; \mathrm{t}(304)=2.33, p=0.020)$, channel 14 at times $3(\mathrm{M}=0.035 \mathrm{SE}=0.015 ; \mathrm{t}(420)=2.41$, $p=0.017)$ and $4(\mathrm{M}=0.051 \mathrm{SE}=0.015 ; \mathrm{t}(420)=3.52$, $p<0.001)$, and channel 15 at times $3(\mathrm{M}=0.039$
$\mathrm{SE}=0.018 ; \mathrm{t}(536)=2.21, p=0.027)$ and $4(\mathrm{M}=0.042$ $\mathrm{SE}=0.018 ; \mathrm{t}(536)=2.38, p=0.017)$. Marginally significant $\mathrm{HbO}_{2}$ increase was found for channels four at time five $(\mathrm{M}=0.026, \mathrm{SE}=0.014 ; \mathrm{t}(536)=1.76, p=0.079)$, channel ten at time three $(M=0.035, S E=0.019 ; \mathrm{t}(536)=1.88$, $p=0.061)$, and channel 17 at time five $(M=0.033$ $\mathrm{SE}=0.018 ; \mathrm{t}(306)=1.85, p=0.067)$.

Additionally, significant $\mathrm{Hbb}$ decrease was found for channel 13 at time $4(M=-0.039$, SE $=0.020$;

Table 3. Significant $\mathrm{HbO2}$ responses to discriminative and affective touch between $7 \mathrm{M}$ and $12 \mathrm{M}$.

\begin{tabular}{|c|c|c|c|c|c|c|c|c|c|}
\hline \multicolumn{5}{|c|}{ Discriminative $7 \mathrm{M}>$ Discriminative $12 \mathrm{M}$} & \multicolumn{5}{|c|}{ Affective $7 \mathrm{M}>$ Affective $12 \mathrm{M}$} \\
\hline Channel & Time Window & $\beta$ & SE & $p$ value & Channel & Time Window & $\beta$ & SE & $p$ value \\
\hline \multirow[t]{2}{*}{2} & 3 & 0.056 & 0.024 & 0.018 & \multirow{5}{*}{\multicolumn{5}{|c|}{ Non-significant results }} \\
\hline & 4 & 0.053 & 0.024 & 0.025 & & & & & \\
\hline 5 & 3 & 0.033 & 0.016 & 0.050 & & & & & \\
\hline 8 & 3 & 0.035 & 0.015 & 0.019 & & & & & \\
\hline 13 & 2 & 0.035 & 0.014 & 0.053 & & & & & \\
\hline \multicolumn{5}{|c|}{ Discriminative $12 \mathrm{M}>$ Discriminative $7 \mathrm{M}$} & \multicolumn{5}{|c|}{ Affective $12 \mathrm{M}>$ Affective $7 \mathrm{M}$} \\
\hline Channel & Time Window & $\beta$ & SE & $p$ value & Channel & Time Window & $\beta$ & SE & $p$ value \\
\hline 6 & 1 & 0.022 & 0.01 & 0.045 & 8 & 4 & 0.033 & 0.015 & 0.025 \\
\hline 14 & 5 & 0.036 & 0.014 & 0.012 & 10 & 3 & 0.046 & 0.019 & 0.017 \\
\hline \multirow[t]{6}{*}{17} & 4 & 0.055 & 0.02 & 0.008 & 11 & 1 & 0.058 & 0.024 & 0.016 \\
\hline & 5 & 0.046 & 0.018 & 0.012 & & 2 & 0.056 & 0.024 & 0.02 \\
\hline & & & & & 14 & 3 & 0.035 & 0.015 & 0.017 \\
\hline & & & & & & 4 & 0.051 & 0.015 & $<0.001$ \\
\hline & & & & & 15 & 3 & 0.039 & 0.018 & 0.027 \\
\hline & & & & & & 4 & 0.042 & 0.018 & 0.017 \\
\hline
\end{tabular}

a Time window 1: 0-5 seg; time window 2: 5-10 seg; time window 3: 10-15 seg; time window 4: 15-20 seg.

bChannels 2, 5 and 8 are located in somatosensory region; channels 10,11,14, 15 and 17 are located in the temporal region.

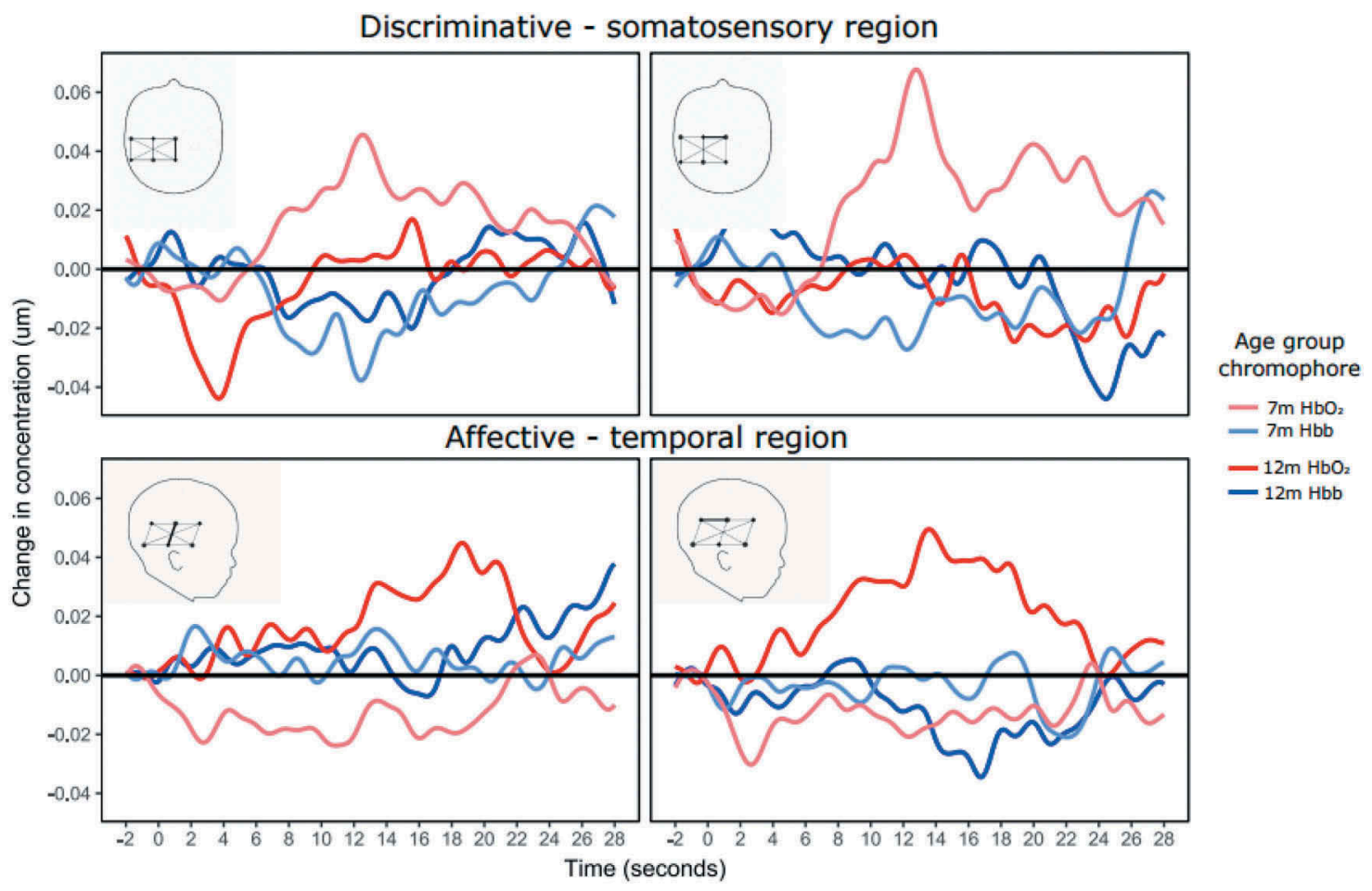

Figure 3. Hemodynamic response function for seven- and 12-month-olds in response to affective and discriminative stimuli. The top panel refers to hemodynamic response function recorded from channels one and two (placed over the somatosensory region) for discriminative touch; the bottom panel refers to hemodynamic response function recorded from channels 14 and 15 (placed over the pSTS region) for affective touch. 
$\mathrm{t}(536)=-1.92, p=0.055)$ and marginally $\mathrm{Hbb}$ decrease was found in channel 1 at time $4(M=-0.030, S E=0.017$; $\mathrm{t}=(536)=-1.8, p=0.072)$.See Table 3 for channelby-channel contrasts between seven- and 12-montholds. See Table S4 in supplementary materials for significant channel-by-channel $\mathrm{Hbb}$ contrasts. Figure 3 displays the hemodynamic response function for the seven- and 12-month-olds for the two conditions in channels placed in the somatosensory and temporal regions.

\section{Discussion}

The current study examined the developmental trajectory of brain responses to affective and discriminative touch by longitudinally following infants from seven to 12 months of age. The brain's hemodynamic activity was analyzed in response to two distinct stimuli applied to the infant's forearm: a discriminative stimulus consisting of a wood block that would target $A \beta$ fibers and an affective stimulus consisting of slow brush strokes targeting CT fibers. Results showed that sevenmonth-old infants process both types of stimuli in the somatosensory cortex. However, at 12 months of age, infants showed a shift by recruiting a posterior region of the temporal lobe (i.e., pSTS - often associated with the processing of socially relevant stimuli) in response to processing affective touch. Moreover, no such activation was found for the seven-month-olds. Another interesting finding was that seven-month-olds presented greater activation to discriminative stimulus in the somatosensory region when compared to the 12month-olds.

The activation of the ipsilateral temporal region to affective touch in 12-month-old infants replicates findings with adult samples that have identified pSTS as one of the brain's regions that is consistently activated following slow stroking of the arm (Bennett et al., 2014; Bjornsdotter et al., 2014; Gordon et al., 2013; Kaiser et al., 2016; Voos et al., 2013). According to the affective-motivational role of the CT fibers (Morrison et al., 2010), the posterior temporal region is more responsive to hairy skin versus glabrous skin (Bjornsdotter et al., 2014; Gordon et al., 2013) and to slower over faster strokes (Voos et al., 2013). In infant populations, the research is somewhat inconclusive. Whereas some studies suggest that this brain region is recruited since very early stages of development (one to two months of age) (Jönsson et al., 2017; Tuulari et al., 2017), others propose it is not until the second semester of life that the pSTS is recruited to process affective touch (Kida \& Shinohara, 2013; Miguel et al., 2017). Jonsson's and Tuulari's studies used measures that were able to evaluate deeper brain regions, like the insula, whereas our study, as well as Kida and Shinoara's, only measured superficial brain regions. It could be that processing of affective touch at a brain level follows a developmental trajectory, maturing earlier in insula and later in the temporal region. Although Jönsson et al. (2017) and Tuulari et al. (2017) also found activation in the temporal region for affective touch, it resulted in a much weaker response compared to the insula. This would be consistent with the fact that associative highorder brain regions, including the temporal lobe, mature later in development (Lenroot \& Giedd, 2006).

In our study, there was a significant difference between the recruitment of the temporal region for the processing of affective touch at seven and at 12 months of age. We can speculate that this shift is congruent with the development of other socially relevant behaviors starting also between nine and 12 months of age, such as joint attention, social referencing, attachment, and implicit mental state attribution (Happe \& Frith, 2014; Johnson et al., 2005). However, more research needs to be conducted, including investigations that look into bilateral responses (left and right pSTS), to fully understand the recruitment of this brain region for the processing of affective touch.

Both age cohorts presented an increased hemodynamic response in optodes placed over the contralateral somatosensory cortex for the two stimuli, confirming that the somatosensory network is recruited to process information sent from both $A \beta$ and $C T$ fibers (Morrison, 2016). Hemodynamic responses of the contralateral somatosensory region to tactile stimuli can be recorded as early as 28 weeks gestation (Bartocci, Bergqvist, Lagercrantz, \& Anand, 2006; Verriotis et al., 2016), suggesting that somatosensory networks are already functional prenatally. In fact, at birth, neural dendritic development, synaptogenesis, and myelination in the somatosensory cortex are already in place (de Graaf-Peters \& Hadders-Algra, 2006; Nevalainen, Lauronen, \& Pihko, 2014; Zecevic \& Rakic, 1991). In terms of affective touch, at five years of age, children present activation in contralateral $\mathrm{S} 1$ and $\mathrm{S} 2$ in response to slow stroking of the forearm (Bjornsdotter et al., 2014), and these activations are similar in adolescents and adults (Gordon et al., 2013; Kaiser et al., 2016; Morrison, 2016).

Interestingly, we found a developmental trajectory for the process of the two types of stimuli; whereas sevenmonth-olds presented a greater activation for channels placed over the somatosensory region for discriminative touch, 12-month-olds presented an increased hemodynamic activity for the channels placed over the temporal region for affective touch. Discriminative touch is transduced by low-threshold mechanoreceptors that transduce information rapidly and allow us to immediately 
identify stimuli in the environment, which can be interpreted as a primary system important for survival purposes (McGlone et al., 2014). It is possible that at seven months of age, when infants are more dependent on their mothers, they are more prone to identify tactile stimuli in the environment and, consequently, present greater activation in the primary sensory region for this stimulus compared to older infants.

From seven to 12 months of age, infants face enormous developmental acquisitions in various domains, including motor and emotional (Valla, Birkeland, Hofoss, \& Slinning, 2017). It is during this period that infants start to move independently and a group of important social capacities emerge, namely joint attention (Mundy et al., 2007), social referencing (Lin \& Green, 2009), and attachment (Minagawa-Kawai et al., 2009). Given the socialaffective specificity of CT afferents (Morrison et al., 2010), it would not be surprising if there was a shift from the neural resources of processing discriminative touch in the sensory region to processing affective touch by recruiting the pSTS. The pSTS region matures later than the somatosensory-cortex (Lenroot \& Giedd, 2006) and has been shown to be susceptible to individual differences in various domains, including affective touch processing (Bennett et al., 2014; Bjornsdotter et al., 2014; Davidovic, Jonsson, Olausson, \& Bjornsdotter, 2016; Voos et al., 2013). The STS presents distinct anatomical and functional features that support a developmental shift in the processing of affective touch. Not only is STS the only anatomical structure that is asymmetric at birth, with the right STS being deeper than the left (Glasel et al., 2011), but the temporal lobe undergoes an increasingly rapid maturation in the first years of life (Bonte et al., 2013; Tanaka, Matsui, Uematsu, Noguchi, \& Miyawaki, 2012), becoming gradually more specialized and focal (Bonte et al., 2013), suggesting that its function is greatly predisposed by individual differences.

To the best of our knowledge, this is the first study exploring the developmental trajectory of the brain's response activity to affective and discriminative touch using a longitudinal design. However, there are several challenges and methodological constraints important to note. A major challenge when studying awake infants is the attrition rate. Although we were able to maintain an attrition rate of $22 \%$ in the seven-month-olds (lower than what is reported in the literature), at 12-month-olds we recruited underwent an increased attrition rate (Lloyd-Fox et al., 2016), resulting in a smaller sample. To deal with non-compliant infants, future studies should include more than one fNIRS session and larger sample sizes (Lloyd-Fox et al., 2016).

Another important aspect concerns the type of baseline stimuli. Since there cannot be a true baseline (no stimuli) for a touch paradigm in infants, we chose to incorporate a silent movie (Fairhurst et al., 2014). This stimulus was chosen to capture the infants' attention and increase the quiet period during data collection, but we do not know the degree of interference of such a task with the hemodynamic signal. Therefore, future studies should test the use of visual and auditory stimuli that are potentially more appealing and control their impact on the hemodynamic response.

A further chief limitation is that we cannot exclusively target $C T$ afferents, since $A \beta$ fibers are present in both glabrous and hairy skin. CT afferents have been typically examined by manipulating the velocity of the stimuli or the site of stimulation (arm versus hand), but this would be hard to implement in infants without introducing more noise in the process of signal acquisition. This was the reason for opting for the use of a stimulus that would target mainly the discriminative component of touch (i.e., a wood block). Finally, the limited number of channels of our system limits the number of cortical regions examined (e.g., hemispheric laterality activations). Future studies should include channels that cover ROI in both hemispheres.

Since Harlow first observed (Harlow \& Zimmermann, 1959) that a newborn rhesus monkey deprived from a monkey mother preferred tactile comfort over nutrition, the importance of physical contact to proper social and emotional development have been extensively documented. Innumerous studies have documented that, in contrast with a more low-level, sensory-perceptual processing of touch, affective touch is also processed in high-order areas of the brain related to the process of social-emotional information, namely the insula, PSTS, ACC, and pre-frontal regions. This study contributed to a better understanding of the developmental trajectory of affective touch in the infant brain, as well as informing about discriminative touch processing. In the future, it would be interesting to include a broader age range, namely newborns, and follow them longitudinally at important developmental milestones.

\section{Research highlights}

- fNIRS captures brain activity in response to discriminative and affective touch.

- Seven-month-olds process discriminative and affective touch in a sensory discriminative region of the brain (somatosensory cortex).

- Twelve-month-old's process affective touch in associative regions of the brain (superior temporal sulcus), similarly to children and adults. 
- Affective touch seems to follow a developmental trajectory that is consistent with the emergence of other social-emotional processes.

\section{Disclosure statement}

No potential conflict of interest was reported by the authors.

\section{Funding}

This study was supported by the Portuguese Science Foundation through an individual doctoral grant to Helga Miguel (SFRH/BD/ 86694/2012). The work was conducted at Psychology Research Center (UID/PSI/01662/2013), University of Minho, and supported by the Portuguese Science Foundation and the Portuguese Ministry of Science, Technology and Higher Education through national funds (PSI/01662) and co-financed by FEDER through COMPETE2020 under the PT2020 Partnership Agreement (POCI01-0145-FEDER-007653); Fundação para a Ciência e a Tecnologia [SFRH/BD/86694/2012].

\section{ORCID}

Helga O. Miguel (D) http://orcid.org/0000-0002-0441-3158

Óscar F. Gonçalves (D) http://orcid.org/0000-0003-2735-9155

\section{References}

Abraira, V. E., \& Ginty, D. D. (2013). The sensory neurons of touch. Neuron, 79(4), 618-639.

Bartocci, M., Bergqvist, L. L., Lagercrantz, H., \& Anand, K. J., 109-117. (2006). Pain activates cortical areas in the preterm newborn brain. Pain, 122, 1-2.

Bennett, R. H., Bolling, D. Z., Anderson, L. C., Pelphrey, K. A., \& Kaiser, M. D. (2014). fNIRS detects temporal lobe response to affective touch. Soc Cogn Affect Neurosci, 9(4), 470-476.

Bjornsdotter, M., Gordon, I., Pelphrey, K. A., Olausson, H., \& Kaiser, M. D. (2014). Development of brain mechanisms for processing affective touch. Front Behav Neurosci, 8, 24.

Bonte, M., Frost, M. A., Rutten, S., Ley, A., Formisano, E., \& Goebel, R. (2013). Development from childhood to adulthood increases morphological and functional inter-individual variability in the right superior temporal cortex. Neuroimage, 83, 739-750.

Bremner, A. J., \& Spence, C. (2017). The development of tactile perception. Adv Child Dev Behav, 52, 227-268.

Cooper, R. J., Selb, J., Gagnon, L., Phillip, D., Schytz, H. W., Iversen, H. K., \& Boas, D. A. (2012). A systematic comparison of motion artifact correction techniques for functional near-infrared spectroscopy. Front Neurosci, 6, 147.

Davidovic, M., Jonsson, E. H., Olausson, H., \& Bjornsdotter, M. (2016). Posterior superior temporal sulcus responses predict perceived pleasantness of skin stroking. Front Hum Neurosci, 10, 432.

de Graaf-Peters, V. B., \& Hadders-Algra, M. (2006). Ontogeny of the human central nervous system: What is happening when? Early Human Development, 82(4), 257-266.

Delpy, D. T., Cope, M., van der Zee, P., Arridge, S., Wray, S., \& Wyatt, J. (1988). Estimation of optical pathlength through tissue from direct time of flight measurement. Phys Med Biol, 33(12), 1433-1442.

Duncan, A., Meek, J. H., Clemence, M., Elwell, C. E., Tyszczuk, L., Cope, M., \& Delpy, D. T. (1995). Optical pathlength measurements on adult head, calf and forearm and the head of the newborn infant using phase resolved optical spectroscopy. Phys Med Biol, 40(2), 295-304.

Essick, G. K., McGlone, F., Dancer, C., Fabricant, D., Ragin, Y., Phillips, N., \& Guest, S. (2010). Quantitative assessment of pleasant touch. Neurosci Biobehav Rev, 34(2), 192-203.

Everdell, N. L., Gibson, A. P., Tullis, I. D. C., Vaithianathan, T., Hebden, J. C., \& Delpy, D. T. (2005). A frequency multiplexed near-infrared topography system for imaging functional activation in the brain. Review of Scientific Instruments, 76, 093705.

Fairhurst, M. T., Loken, L., \& Grossmann, T. (2014). Physiological and behavioral responses reveal 9-month-old infants' sensitivity to pleasant touch. Psychol Sci, 25(5), 1124-1131.

Field, T. (2001). Touch. MA: Cambridge.

Fifer, W. P., \& Moon, C. (2003). Prenatal development. An introduction to developmental psychology (pp. pp. 95-114). Oxford, UK: Blackwell Publishing.

Gallace, A., \& Spence, C. (2010). The science of interpersonal touch: An overview. [Review]. Neuroscience \& Biobehavioral Reviews, 34(2), 246-259.

Glasel, H., Leroy, F., Dubois, J., Hertz-Pannier, L., Mangin, J. F., \& Dehaene-Lambertz, G. (2011). A robust cerebral asymmetry in the infant brain: The rightward superior temporal sulcus. Neuroimage, 58(3), 716-723.

Gordon, I., Voos, A. C., Bennett, R. H., Bolling, D. Z., Pelphrey, K. A., \& Kaiser, M. D. (2013). Brain mechanisms for processing affective touch. Hum Brain Mapp, 34(4), 914-922.

Happe, F., \& Frith, U. (2014). Annual research review: Towards a developmental neuroscience of atypical social cognition. [Review]. J Child Psychol Psychiatry, 55(6), 553-557.

Harlow, H. F., \& Zimmermann, R. (1959). Affectional responses in the infant monkey. Science, 130, 421-431.

Hertenstein, M. J., Verkamp, J. M., Kerestes, A. M., \& Holmes, R. M. (2006). The communicative functions of touch in humans, nonhuman primates, and rats: A review and synthesis of the empirical research. Genet Soc Gen Psychol Monogr, 132(1), 5-94.

Hooker, D. (1952). The prenatal orgin of behavior. Lawrence, KS: University of Kansas Press.

Humphrey, T. (1964). Some correlations between the apperance of human fetal reflexes and the development of nervous system. Progress in Brain Research, 4, 93-135.

Jean, A. D. L., \& Stack, D. M. (2012). Full-term and very-lowbirth-weight preterm infants' self-regulating behaviors during a Still-Face interaction: Influences of maternal touch. Infant Behav Dev, 35(4), 779-791.

Jean, A. D. L., Stack, D. M., \& Fogel, A. (2009). A longitudinal investigation of maternal touching across the first 6 months of life: Age and context effects. Infant Behav Dev, 32(3), 344349.

Johnson, M. H., Griffin, R., Csibra, G., Halit, H., Farroni, T., de Haan, M., \& Richards, J. (2005). The emergence of the social brain network: Evidence from typical and atypical development. Dev Psychopathol, 17(3), 599-619.

Jönsson, E. H., Kotilahti, K., Heiskala, J., Wasling, H. B., Olausson, H., Croy, I., ... Nissilä, I. (2017). Affective and non-affective touch evoke differential brain responses in 2-month-old infants. Neuroimage, 169, 162-171. 
Jurcak, V., Tsuzuki, D., \& Dan, I. (2007). 10/20, 10/10, and 10/5 systems revisited: Their validity as relative head-surfacebased positioning systems. Neuroimage, 34(4), 1600-1611.

Kaiser, M. D., Yang, D. Y., Voos, A. C., Bennett, R. H., Gordon, I., Pretzsch, C., \& Pelphrey, K. A. (2016). Brain mechanisms for processing affective (and nonaffective) touch are atypical in autism. Cereb Cortex, 26(6), 2705-2714.

Kandel, E. R., Schawartz, J. H., \& Jessell, T. M. (2000). Principles of neural science. New York: McGraw-Hill Companies.

Kida, T., \& Shinohara, K. (2013). Gentle touch activates the prefrontal cortex in infancy: An NIRS study. Neuroscience Letters, 541, 63-66.

Lenroot, R. K., \& Giedd, J. N. (2006). Brain development in children and adolescents: Insights from anatomical magnetic resonance imaging. [Review]. Neuroscience \& Biobehavioral Reviews, 30(6), 718-729.

Leppanen, J. M., \& Nelson, C. A. (2009). Tuning the developing brain to social signals of emotions. Nat Rev Neurosci, 10(1), 3747.

Lin, H.-C., \& Green, J. A. (2009). Infants' expressive behaviors to mothers and unfamiliar partners during face-to-face interactions from 4 to 10 months. Infant Behav Dev, 32(3), 275-285.

Lloyd-Fox, S., Begus, K., Halliday, D., Pirazzoli, L., Blasi, A., Papademetriou, M., \& Elwell, C. E. (2016). Cortical specialisation to social stimuli from the first days to the second year of life: A rural Gambian cohort. Dev Cogn Neurosci. doi:10.1016/j.dcn.2016.11.005

Lloyd-Fox, S., Blasi, A., \& Elwell, C. (2010). Illuminating the developing brain: The past, present and future of functional near infrared spectroscopy. Neuroscience \& Biobehavioral Reviews, 34(3), 269-284. doi:10.1016/j.neubiorev.2009.07.008

Lloyd-Fox, S., Szeplaki-Kollod, B., Yin, J., \& Csibra, G. (2015). Are you talking to me? Neural activations in 6-month-old infants in response to being addressed during natural interactions. Cortex, 70, 35-48.

Lloyd-Fox, S., Wu, R., Richards, J. E., Elwell, C. E., \& Johnson, M. H. (2015). Cortical activation to action perception is associated with action production abilities in young infants. Cereb Cortex, 25(2), 289-297.

Loken, L. S., Wessberg, J., Morrison, I., McGlone, F., \& Olausson, H. (2009). Coding of pleasant touch by unmyelinated afferents in humans. Nat Neurosci, 12(5), 547-548.

Mcglone, F., Vallbo, A. B., Olausson, H., Loken, L., \& Wessberg, J. (2007). Discriminative touch and emotional touch. Canadian Journal of Experimental Psychology/revue Canadienne De Psychologie Expérimentale, 61(3), 173-183. doi:10.1037/cjep2007019

McGlone, F., Wessberg, J., \& Olausson, H. (2014). Discriminative and affective touch: Sensing and feeling. Neuron, 82(4), 737-755.

Miguel, H. O., Lisboa, I. C., Gonçalves, O. F., \& Sampaio, A. (2017). Brain mechanisms for processing discriminative and affective touch in 7-month-old infants. Dev Cog Neurosci, 17, 30013-0. doi:10.1016/j.den.2017.10.008

Minagawa-Kawai, Y., Matsuoka, S., Dan, I., Naoi, N., Nakamura, K., \& Kojima, S. (2009). Prefrontal activation associated with social attachment: Facial-emotion recognition in mothers and infants. Cerebral Cortex, 19(2), 284-292.

Morrison, I. (2016). ALE meta-analysis reveals dissociable networks for affective and discriminative aspects of touch. Hum Brain Mapp, 37(4), 1308-1320.
Morrison, I., Loken, L. S., \& Olausson, H. (2010). The skin as a social organ. [Review]. Exp Brain Res, 204(3), 305-314.

Mountcastle, V. B. (2005). The sensory hand. Cambridge: Harvard University Press.

Mundy, P., Block, J., Delgado, C., Pomares, Y., Van Hecke, A. V., \& Parlade, M. V. (2007). Individual differences and the development of joint attention in infancy. Child Development, 78(3), 938-954.

Nevalainen, P., Lauronen, L., \& Pihko, E. (2014). Development of human somatosensory cortical functions - What have we learned from magnetoencephalography: A review. [Review]. Front Hum Neurosci, 8, 158.

Olausson, H., Lamarre, Y., Backlund, H., Morin, C., Wallin, B. G., Starck, G., \& Bushnell, M. C. (2002). Unmyelinated tactile afferents signal touch and project to insular cortex. Nat Neurosci, 5(9), 900-904.

Olausson, H., Wessberg, J., Morrison, I., McGlone, F., \& Vallbo, Å. (2010). The neurophysiology of unmyelinated tactile afferents. Neuroscience \& Biobehavioral Reviews, 34(2), 185-191.

Olausson, H. W., Cole, J., Vallbo, A., McGlone, F., Elam, M., Kramer, H. H., \& Bushnell, M. C. (2008). Unmyelinated tactile afferents have opposite effects on insular and somatosensory cortical processing. Neurosci Lett, 436(2), 128-132.

Ravicz, M. M., Perdue, K. L., Westerlund, A., Vanderwert, R. E., \& Nelson, C. A. (2015). Infants' neural responses to facial emotion in the prefrontal cortex are correlated with temperament: A functional near-infrared spectroscopy study. Front Psychol, 6, 922.

Tanaka, C., Matsui, M., Uematsu, A., Noguchi, K., \& Miyawaki, T. (2012). Developmental trajectories of the fronto-temporal lobes from infancy to early adulthood in healthy individuals. Dev Neurosci, 34(6), 477-487.

Tomasello, M., Carpenter, M., \& Liszkowski, U. (2007). A new look at infant pointing. Child Development, 78(3), 705-722.

Triscoli, C., Olausson, H., Sailer, U., Ignell, H., \& Croy, I. (2013). CT-optimized skin stroking delivered by hand or robot is comparable. Front Behav Neurosci, 7, 208.

Tuulari, J. J., Scheinin, N. M., Lehtola, S., Merisaari, H., Saunavaara, J., Parkkola, R., ... Björnsdotter, M. (2017). Neural correlates of gentle skin stroking in early infancy. Dev Cog Neurosci, 17, 30082-30088.

Valla, L., Birkeland, M. S., Hofoss, D., \& Slinning, K. (2017). Developmental pathways in infants from 4 to 24 months. Child Care Health Dev, 43(4), 546-555.

Van de Winckel, A., Klingels, K., Bruyninckx, F., Wenderoth, N., Peeters, R., Sunaert, S., \& Feys, H. (2013). How does brain activation differ in children with unilateral cerebral palsy compared to typically developing children, during active and passive movements, and tactile stimulation? An fMRI study. Res Dev Disabil, 34(1), 183-197.

Verriotis, M., Fabrizi, L., Lee, A., Cooper, R. J., Fitzgerald, M., \& Meek, J. (2016). Mapping cortical responses to somatosensory stimuli in human infants with simultaneous near-infrared spectroscopy and event-related potential recording. eNeuro, 3(2). doi:10.1523/ENEURO.0026-16.2016

Voos, A. C., Pelphrey, K. A., \& Kaiser, M. D. (2013). Autistic traits are associated with diminished neural response to affective touch. Soc Cogn Affect Neurosci, 8(4), 378-386.

Zecevic, N., \& Rakic, P. (1991). Synaptogenesis in monkey somatosensory cortex. Cereb Cortex, 1(6), 510-523. 